\title{
Endoscopic fenestration of a symptomatic esophageal duplication cyst
}

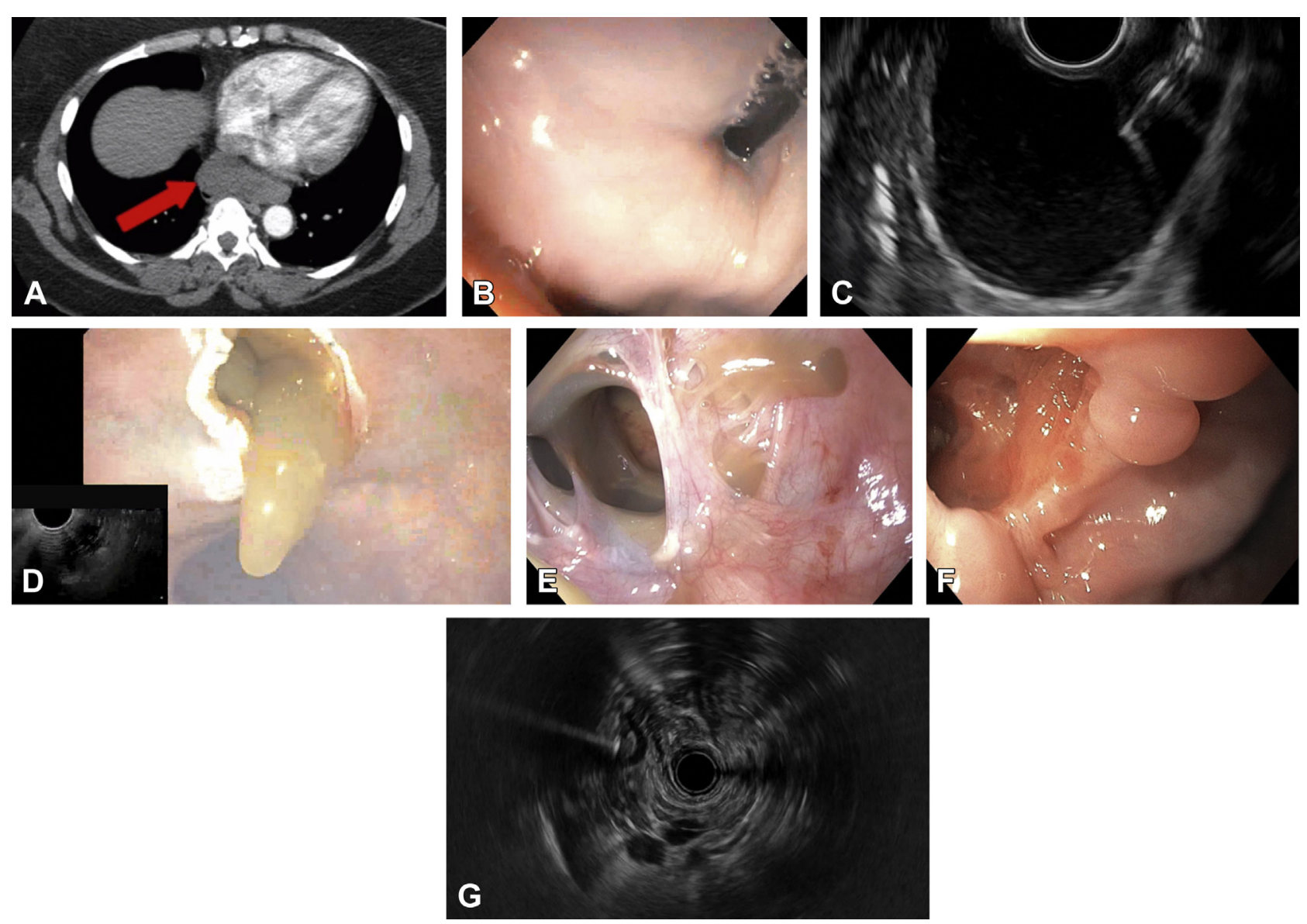

Figure 1. A, CT scan of the chest demonstrating $6.8 \times 3.2 \mathrm{~cm}$ esophageal mass. B, Upper endoscopic view showing mass effect in the distal esophagus just above the lower-esophageal sphincter. C, EUS view of electrocautery knife incision into the distal esophagus. D, Endoscopic view following cyst puncture. E, View from within the cyst cavity following lavage. F, Follow-up endoscopy showing simple diverticulum. G, Follow-up EUS showing resolution of cyst.

A 52-year-old woman with a medical history of GERD and hypertension presented to her primary care doctor with several years of substernal chest pressure, which had gradually worsened over the preceding 2 to 3 months. Initial workup for a cardiac cause of her symptoms was negative. She underwent contrast-enhanced CT scan of the chest, which demonstrated a $6.8 \times 3.2$ $\mathrm{cm}$ mass in the posterior mediastinum, which was compliant with the distal esophagus (Fig. 1A). Upper endoscopy with EUS was used to better characterize the mass and demonstrated an anechoic cyst between the left atrium and the esophagus; the cyst was clearly associated with the esophageal wall. Doppler US demonstrated a lack of blood flow through the cyst. The use of Doppler US allowed for visualization of interposing vessels, which were absent in this case, and would have increased the risk of bleeding associated with endoscopic intervention.

After discussion of the case at thoracic tumor board and in consideration of the unequivocally benign appearance of the cyst, it was decided that the preferred management was for the least-invasive therapy possible. The patient was referred for endoscopic management and, before the procedure, received 
intravenous antibiotics to decrease the risk of periprocedural infection.

On upper-endoscopic view, an obvious mass effect from the cyst was seen in the distal esophagus, just above the lower-esophageal sphincter (Fig. 1B). An electrocautery knife was used to make an incision into the distal esophagus, with EUS serving to guide instrument placement (Fig. 1C). A hook knife was selected to create a larger opening into the cyst and decrease the risk of abscess formation. After incision into the cyst, copious mucinous fluid drained into the esophageal lumen (Fig. 1D). The upper endoscope was then reinserted and was used to view the inside of the cystic cavity (Fig. 1E). The cavity was lavaged with saline solution and $\mathrm{N}$ acetylcysteine to clear the cavity. Biopsy specimens were taken from the lining of the cyst cavity with standard biopsy forceps. The use of endoclips at the base of the incision was critical to prevent further dissection of the esophageal lumen, which could have led to perforation.

Histopathologic staining of the biopsy tissue demonstrated pseudostratified, ciliated, columnar epithelium, which was consistent with an esophageal duplication cyst. Upper endoscopy with EUS was repeated 4 weeks after the initial procedure, and the site of the former cyst was now demonstrated to be a simple diverticula (Fig. 1F). Repeated EUS demonstrated complete resolution of the fluid-filled cyst (Fig. 1G). The entire procedure was completed on an outpatient basis, and there were no adverse events.

Esophageal duplication cysts are the embryologic remnant of the primitive diverticula, which normally forms the dorsal esophagus and ventral respiratory tract. They are benign mediastinal masses that usually present in infancy or in childhood and involve the distal third of the esophagus.

Endoscopic fenestration by a skilled therapeutic endoscopist is safe and efficacious in the management of these cysts. The approach allows for an outpatient procedure with decreased postoperative recovery time. The endoscopic cavity should be completely debrided to decrease the risk of infection after the procedure, and the base of the cyst should be clipped to prevent spontaneous dissection and perforation of the esophagus. Previous case reports have described resection or banding of esophageal duplication cysts; however, this is the first video case report (Video 1, available online at www.VideoGIE.org) to describe endoscopic fenestration in the management of these cysts.

\section{DISCLOSURE}

Dr Grimm is a consultant for Boston Scientific. Dr Baron is the recipient of research grants from Boston Scientific, ConMed, Cook Medical, Gore Medical, and Olympus and is a consultant and paid speaker for Cook Endoscopy. The other author disclosed no financial relationships relevant to this publication.

Theodore W. James, MD, lan S. Grimm, MD, Todd H. Baron, MD, University of North Carolina, Chapel Hill, North Carolina, USA

Copyright (c) 2017 American Society for Gastrointestinal Endoscopy. Published by Elsevier Inc. This is an open access article under the CC BY-NC-ND license (http://creativecommons.org/licenses/by-nc-nd/4.0/).

http://dx.doi.org/10.1016/j.vgie.2017.04.002 